\title{
Dedicated optical systems of the Institute of Applied Optics
}

\author{
Dariusz Litwin, ' Jacek Galas, Marek Daszkiewicz, Tadeusz Kryszczyński, Adam Czyżewski, Kamil Radziak \\ Department of Physical Optics, Łukasiewicz Research Network-Maksymilian Pluta Institute of Applied Optics, \\ Kamionkowska 18, 03-805 Warsaw, Poland
}

Received June 3, 2019; accepted June 26, 2019; published June 30, 2019

\begin{abstract}
The paper presents a collection of selected optical systems recently developed in the Institute of Applied Optics-INOS. The collection includes the family of techniques where a continuously modified wavelength facilitates high accuracy measurements of optical and geometrical features of the object in question i.e. the variable wavelength interferometry and confocal chromatic sensors. In addition, the paper refers to the construction of a new type of a spectrometer with rotating plasma and an illumination system supporting the road safety.
\end{abstract}

Since 1989 the Institute of Applied Optics (INOS) has been the successor of the Central Laboratory of Optics, which was founded in 1948, and has become the state optical research and development centre for the Polish industry. In 2013 it changed its name to the Maksymilian Pluta Institute of Applied Optics and in 2019 joined the newly established Łukasiewicz Research Network organized in a way similar to TNO-Netherlands Organization for Applied Scientific Research, Fraunhofer-Gesellschaft, and French Carnot Institute ARTS-Actions for Research, Technology and Society (though there are some differences), which strengthened its application and industry-oriented character. This has inevitably directed the authors' professional career and their research interest, to market-oriented issues and entrepreneurs' needs.

Variable wavelength interferometry, called in short VAWI is traditionally a leading optical technology in the Institute [1-5]. A family of techniques (for transmitted and reflected light) is suitable for measuring objects that create optical path difference at the order of a few wavelengths. In such cases the classical approach consisting in measuring deflection of interference fringes is not supportive because of the edge effects that disconnect the integrity of interference fringes. A family of these methods has been proposed originally by Pluta in the 1980s but at that time image processing devices and computers were hardly available. Digital technology unfolded an entirely new approach to classical measurement procedures. The Institute's team has taken that new opportunity and transformed the technique into fully automated measurement devices of commercial quality. In addition, during the realization of these projects the team contributed significantly to method improvement by modifying the existing and developing

\footnotetext{
*E-mail: dlitwin@inos.pl; adlitwin@pro.onet.pl
}

new solutions and algorithms, simultaneously extending the field of application. This has concerned both construction aspects of the systems and software development in the context of creating computerized instruments [6-8]. The VAWI collection of instruments constitutes now the core of the INOS commercial offer. It can be applicable in an industrial environment for measuring textile and optical fibres, strips of thin films, wave plates retardation and nonlinear effects. A detailed description of the approach is beyond the scope of this paper but can be found in other publications listed below. The technique consists in monitoring and measuring the change of phase in the fringe field during a continuously varying wavelength of the light source. The first systems were not supported by a computer and therefore all operations and following calculations had to be performed manually, which was a tedious and long procedure- not very attractive from a commercial point of view. The researcher had to watch the moving fringe field and identify selected, easy to recognize phase values i.e. minima and maxima of fringes and write down the corresponding wavelengths. In the VAWI methodology these characteristic locations were called 'coincidences' since they refer to an equal phase of the fringe pattern in the empty fringe field and in the object region. In other VAWI methods these coincidences occur between specially selected reference lines and the fringes. In automated systems coincidences are replaced by more efficient registration of phases for subsequent corresponding wavelengths.

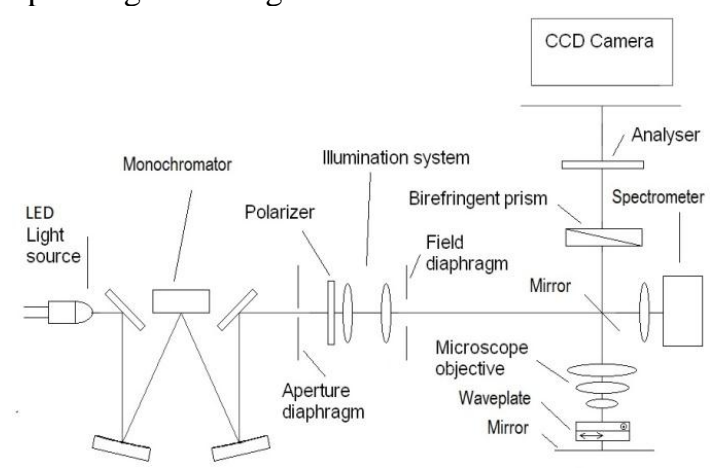

Fig. 1. Optical setup of the VAWI interferometer in reflected-light mode. 


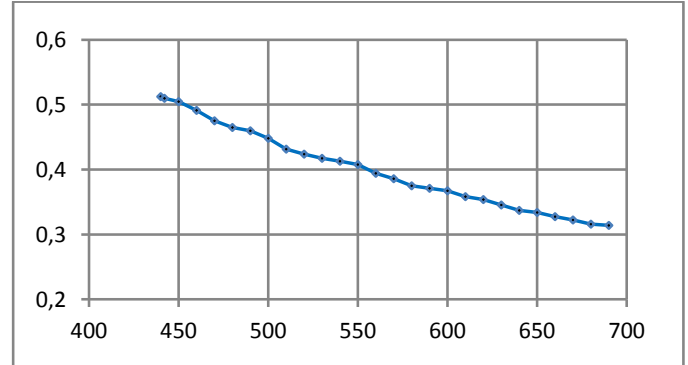

Fig. 2. Optical path deference [parts of wavelengths] vs wavelength [nm].

The description of the VAWI systems, including their non-standard versions [9], can be found in the mentioned literature. In the context of industrial applications it is worth mentioning a new system arranged in reflectedlight mode [10] to be used for measuring the retardation of wave-plates [11], which is a crucial chain in production process quality control (Fig.1). The advantage of the instrument is that the light passes the measured plate twice and the inter-fringe distance is twice smaller compared to the transmitted-light system, which together creates the potential for higher accuracy. An exemplary optical path difference plot is presented in Fig. 2. The current accuracy is well above $\lambda / 500$ and there is still room for improvement in mechanical/optics design as well as in software modernization.

A new measurement challenge emerged in the context of developing a microscope with a focused beam of neutral helium atoms of low energy ("INA" - EU project) [12]. The beam was used as an imaging probe for noninvasive surface inspecting and analysis in different branches of science and industry. The probe was focused onto the sample by a single silicon wafer deformed electrostatically. The exact shape of the wafer was modeled by a system of electrodes precisely controlled by dedicated software based on finite element approach. The required precision of curvature of the wafer depended on the wafer quality. Therefore, several improvements to the manufacturing process were introduced at macroscopic and atomic levels to improve the initial shape. The EU project encouraged the INOS team to build measuring systems utilizing confocal sensors. They were built for the measurements of flatness and thickness of the wafer without any electric field and then, in cooperation with the University of Graz group (Austria), the shape under the electric field. Thanks to these efforts the technological process of the wafers was enhanced to obtain a highquality atomic mirror [13-14]. The chromatic confocal sensor principle of operation is based on the chromatic aberration of a lens, see Fig. 3. In designing all optical systems great efforts are made to minimize the aberration but in this device, it is arranged otherwise. The light source, which is now usually an LED, is focused on a measured surface by a specialized objective with increased longitudinal chromatic aberration so that the measurement range can be extended. The reflected light is analyzed by a spectrometer. The spectrum maximum of a collected light pattern refers to a certain distance between the head and the measured surface, which needs to be initially calibrated, normally at the manufacturer level.

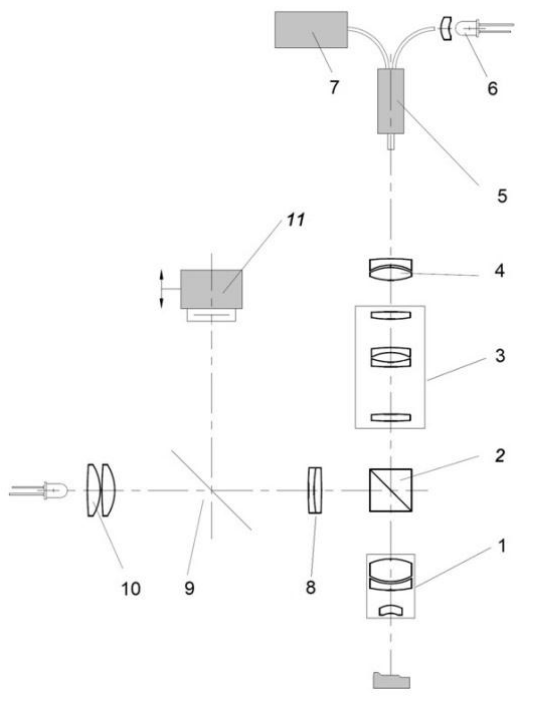

Fig. 3. Confocal head with visual inspecting systems.

The majority of confocal sensors include microscope lenses though they do not provide the ability to observe what is actually measured. In certain applications simultaneously performed measurements with visual inspection of the object, especially significantly larger than the area of the measurement spot of the sensor, are very useful. Therefore, the INOS team designed a specialized confocal head with an integrated visual inspection system equipped with an auxiliary CCD camera or an eyepiece [15]. The system was built as a combination of two light paths. The first path includes the spectrometer and performs measuring (7). The second path is designed for visual inspection of the measured surface. Both paths utilize a single main microscopequality objective (1) with apochromatic correction. The light is divided into these paths by means of a beam splitter (2). The illuminating system has also two branches: for measuring (6) and visual inspection (10). The illuminator (6) with a photocoupler (5) introduces white light into the optical system. The reflected light first collected by the objective and then by the same coupler (5) is directed to the spectrometer. The spectrometric path is additionally equipped with a chromatic quasi-spyglass (3), which is located between the beam splitter (2) and the auxiliary lens (4). It has the same numerical aperture as that of the main objective (1). The chromatic quasispyglass is the only source of the required chromatic 
aberration. The visual path includes a tube lens (8) and an additional beam splitter (9) between the tube objective (8) and the visual illuminator (10). In the visual path the tube lens (8) transmits the image of the object surface to the CCD sensor (11). The camera (11) can be moved within a range of $\pm 20.4 \mathrm{~mm}$ in order to smoothly secure a sharp image of the surface being observed. The measuring range of the described confocal sensor is equal to $2.55 \mathrm{~mm}$ expecting the spectrometer to be in the range 400 to $700 \mathrm{~nm}$.

Another task for the INOS team was the development of an optical emission spectrometer with helium microwave rotating plasma as the excitation source [16]. The approach is a competitive solution to quite wellknown ICP spectrometers. Helium is used as plasma and carrier gases, which potentially increases its ability to determine elements such as halogens and some nonmetals. The new system is expected to offer decreased operating costs considering the flow difference: $1 \mathrm{~L}$ $\mathrm{He} / \mathrm{min}$ compared to about $15 \mathrm{~L} \mathrm{Ar} / \mathrm{min}$ for ICP. The device covers the $165-840 \mathrm{~nm}$ spectral range, sensitivity at the level of ppb while the spectral resolution is equal to $0.01 \mathrm{~nm}$.

In 2007 the INOS team together with the Road And Bridge Research Institute group was included in the project devoted to the design of optical systems for variable message signs. There is no doubt that the surface and vertical traffic signs affect significantly road safety. They must be correctly designed and clearly visible regardless of weather conditions.

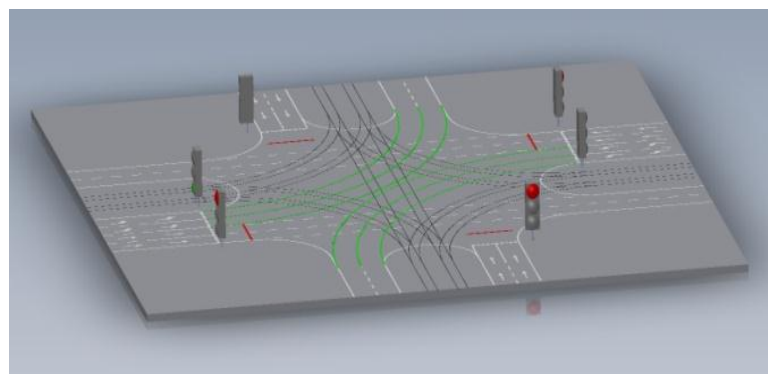

Fig. 4. SPIKer: the green and red dot patterns projected on the road.

The team designed and installed an intelligent, horizontal sign system called the SPIKer [17]. The green part of the system supports finding the most effective path through complex intersections by displaying green dot pattern on the road surface. Especially, the green lanes help motorists in performing left turns in bad weather conditions or at night - Figs. 4 and 5. The red part displays the blocking pattern suggestively discouraging the motorist from entering the intersection at the red light. The system can be also used when the traffic is temporarily reoriented because of accidents or construction works. The lamps are mounted above the intersection and the pattern is effectively displayed on any surface including that covered by snow.

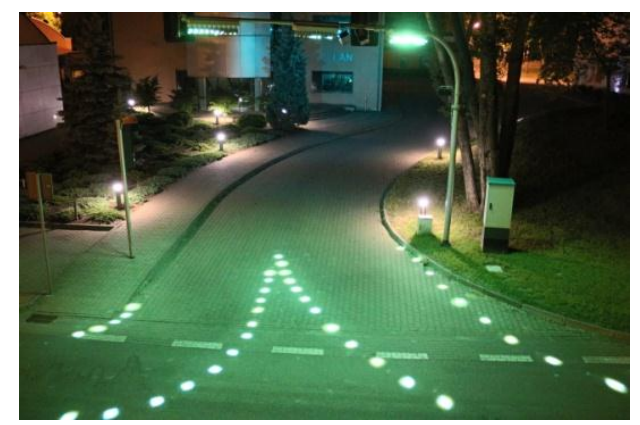

Fig.5. SPIKer system: the green dot pattern displayed on the road.

The VAWI project was partly funded by the Polish Ministry of Education and Science, grant no. 4T07D 00527. The work concerning road safety was funded partly within two projects: "Motorist-friendly intelligent road intersection" (PBS3/B6/29/2015) and "Experimental road signs in the context of the road user behavior" (RIDI/36/5/NCBR/2016). The former project has been supported by the National Centre for Research and Development, Warsaw, Poland - NCBiR (through the Applied Research Programme) the latter jointly by NCBiR and General Directorate for National Roads and Motorways - GDDKiA.

\section{References}

[1] M. Pluta, Advanced Light Microscopy (Vol. 3, PWN, Elsevier, Warszawa-Amsterdam-London-New York-Tokyo, 1993).

[2] M. Pluta, J. Opt. Soc. Am. A4(11), 2107 (1987).

[3] M. Pluta, J. Microscopy 149(2), 97 (1988).

[4] M. Pluta, J. Microscopy 145(2), 191 (1987).

[5] K.A. El-Farahaty, A.M. Sadik, A.M. Hezma, Internat. J. Polymeric Mat. 56(7),715 (2007).

[6] J. Galas, D. Litwin, M. Daszkiewicz, Proc. SPIE 10142, 101421R (2016).

[7] A. Sadik, W.A. Ramadan, D. Litwin, Measur. Scien. Technol., IOP Publishing 14(10), 1753 (2003).

[8] J. Galas, S. Sitarek, D. Litwin, M. Daszkiewicz, Proc. SPIE 10445, 1044504 (2017).

[9] D. Litwin, A.M. Sadik, Opt. Applicata 28(2), 139 (1998).

[10] D. Litwin, J. Galas, N. Błocki, Proc. SPIE 6188, 61880F (2006).

[11] M. Pluta, Opt. Laser Technol. 19(3), 131 (1987).

[12] K. Fladischer et al., New J. Physics 12(3) 033018 (2010).

[13] K. Fladischer et al., Precision Engin. Elsevier 32(3), 182 (2008).

[14] A.E. Weeks et al., Semicond. Scien. Technol., IOP Publishing, 22(9), 997 (2007).

[15] D. Litwin et al., Proc. SPIE 10808, 10 8080B (2018).

[16] D. Litwin et al., Proc. SPIE 10142 101421Q (2016).

[17] D. Litwin et al., Proc SPIE 10142 1014214, (2016). 